\title{
Effect of intravenous iron replacement therapy on exercise capacity in iron deficient anemic patients after cardiac surgery
}

\author{
Cinzia Nugara ${ }^{1,2}$, Giuseppe Vitale ${ }^{1}$, Giuseppa Caccamo ${ }^{1}$, Silvia Sarullo ${ }^{3}$, Francesco Giallauria ${ }^{4}$, \\ Antonino Di Franco 5 , Silvia Vitale ${ }^{6}$, Filippo M. Sarullo ${ }^{1}$ \\ ${ }^{1}$ Cardiovascular Rehabilitation Unit, Buccheri La Ferla Fatebenefratelli Hospital, Palermo, Italy; ${ }^{2}$ Biomedical \\ Department of Internal Medicine and Specialities (DIBIMIS), University of Palermo, IRCSS Bonino Pulejo, Messina, \\ Italy; ${ }^{3}$ University of Palermo, Cardiology Unit, Palermo, Italy; ${ }^{4}$ Department of Translational Medical Sciences, \\ Division of Internal Medicine, Metabolic and Cardiac Rehabilitation Unit, University of Naples Federico II, Italy; \\ ${ }^{5}$ Department of Cardiothoracic Surgery, Weill Cornell Medicine, New York City, NY, USA; ${ }^{6}$ Medical Biotechnology and \\ Molecular Medicine, University of Palermo, Italy
}

\begin{abstract}
Correspondence: Filippo M. Sarullo, Cardiovascular Rehabilitation Unit, Buccheri La Ferla Fatebenefratelli Hospital, Via Messina Marine 197, 90121 Palermo, Italy.

Tel. +39.091.479263 - Fax: +39.09. 477625.

E-mail: fsarullo@neomedia.it

Keywords: Iron deficiency; anemia; cardiac surgery; exercise capacity; ferric carboxymaltose; ferric gluconate.

Contributions: FMS, contribution to work concept; CN, FMS, manuscript drafting; $\mathrm{CN}, \mathrm{GV}, \mathrm{GC}, \mathrm{SS}, \mathrm{SV}$, contribution to data acquisition, analysis, and interpretation; ADF, FG, critically revised the manuscript. All the authors read and approved the final version of the manuscript, and agreed to be accountable for all aspects of the work ensuring integrity and accuracy.
\end{abstract}

Conflict of interest: The authors declare that they have no competing interests, and all authors confirm accuracy.

Availability of data and materials: All data generated or analyzed during this study are included in this published article.

Ethics approval and consent to participate: All procedures performed in studies involving human participants were in accordance with the ethical standards of the institutional and/or national research committee and with the 1964 Helsinki declaration and its later amendments or comparable ethical standards.

Informed consent: Informed consent was not obtained from all individual participants included in the study due to retrospective study design.

Consent for publication: Not applicable.

Received for publication: 3 November 2019.

Accepted for publication: 2 January 2020.

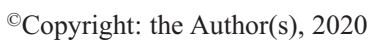

Licensee PAGEPress, Italy

Monaldi Archives for Chest Disease 2020; $90: 1196$

doi: 10.4081/monaldi.2020.1196

This article is distributed under the terms of the Creative Commons Attribution Noncommercial License (by-nc 4.0) which permits any noncommercial use, distribution, and reproduction in any medium, provided the original author(s) and source are credited.

\begin{abstract}
Iron deficiency (ID) is recognized as an important comorbidity in patients undergoing cardiac surgery; however, it still remains under-diagnosed and under-treated in clinical practice. This study aims at comparing efficacy and the effects on exercise capacity of intravenous ferric carboxymaltose (FCM) versus ferric gluconate (FG) in patients with ID anemia (IDA) resulting from cardiac surgery. We retrospectively analyzed data from our records of inhospital patients with IDA after cardiac surgery undergoing cardiac rehabilitation. Group I was treated with FG, group II with FCM. Efficacy measures included changes (baseline $v s$ discharge) in hemoglobin $(\mathrm{Hb})$ and in distance traveled at six-minutes walking test (6MWT). Data from 74 in-patients (mean age $67.5 \pm 10.4$ years, $43 \%$ women) were analyzed. At discharge, patients treated with FCM showed higher levels of $\mathrm{Hb}(11.1 \pm 1.2 \mathrm{~g} / \mathrm{dl} v s 10.2 \pm 1.1 \mathrm{~g} / \mathrm{dl}$; $\mathrm{p}=0.001)$, greater distance traveled at 6MWT $(279.2 \pm 108.8$ meters vs $236.3 \pm 72.7$ meters; $\mathrm{p}=0.048$ ), and lower in-hospital rehabilitation length of stay ( $20.3 \pm 7$ vs $25.3 \pm 11.7$ days; $\mathrm{p}=0.043$ ) as compared to FG group. At multivariate analysis, the most powerful predictors of $\mathrm{Hb}$ increase $>1 \mathrm{~g} / \mathrm{dl}$ at discharge were transferrin levels $(\mathrm{p}=0.019)$ and treatment with FCM $(\mathrm{p}<0.001)$. FCM replacement therapy and iron serum levels were the most powerful predictors of 6MWT distance improvement $(>100$ meters) at discharge $(\mathrm{p}=0.13$ and $\mathrm{p}=0.003$, respectively). In patients with IDA following cardiac surgery, intravenous FCM is effective in restoring $\mathrm{Hb}$ levels and in improving exercise capacity after cardiac surgery.
\end{abstract}

\section{Introduction}

Patients undergoing cardiac surgery are at increased risk of excessive perioperative bleeding and increased blood product transfusion [1]. In the setting of patients undergoing surgery, anemia is present in a third of all patients [2], but it is more prevalent in cardiac surgery where it may be interest about $50 \%$ of patients [3].

Iron deficiency (ID) is increasingly recognized as an important comorbidity in patients undergoing cardiac surgery and it has been shown to be responsible of $29 \%$ of preoperative anemia [1]. ID reduces exercise capacity in patients with chronic heart failure 
(CHF) but also in healthy volunteers [4-6]. Iron plays a crucial role in systemic oxygen $\left(\mathrm{O}_{2}\right)$ delivery and utilization [7]. Iron is also an obligate component of enzymes involved in cellular respiration, oxidative phosphorylation, vascular homeostasis, nitricoxidegeneration, and the citric acid cycle [8]. Hence, cells with high energy demands, including skeletal and cardiomyocytes, are particularly sensitive to iron depletion [9]

The most rapid and effective method of correcting anemia is transfusing red blood cells; however, blood transfusion itself is not riskless [1]. In the specific setting of cardiac surgery, blood transfusions are associated with both infections and ischemic postoperative morbidity, longer hospital stay, increased early and late mortality and greater hospital costs [10]. On the other hand, prophylactic administration of red blood cells to correct anemia preoperatively leads to lower rates of intraoperative anemia and transfusion compared with intraoperative transfusions [11]. Treatment of perioperative anemia using oral or intravenous (IV) iron replacement therapy is safer, cheaper and relatively more convenient than blood transfusion.

The aim of the present study was to compare the efficacy and the impact on exercise capacity of IV ferric carboxymaltose (FCM) versus ferric gluconate (FG) in patients with ID anemia resulting from cardiac surgery.

\section{Methods}

A retrospective analysis of medical records of consecutive adult patients with anemia following cardiac surgery hospitalized, from January 2017 to December 2018, was completed at the Cardiovascular Rehabilitation Unit of Buccheri La Ferla Fatebenefratelli Hospital in Palermo, Italy.

Inclusion criteria were: $\mathrm{Hb} \leq 12 \mathrm{~g} / \mathrm{dl}$, ferritin $\leq 100 \mathrm{ng} / \mathrm{ml}$ (or $\leq 300 \mathrm{mg} / \mathrm{ml}$ if transferring saturation [TSAT] $\leq 20 \%$ ), and left ventricle ejection fraction (LVEF) $>50 \%$. Exclusion criteria were age $<18$ years, pregnancy, iron therapy and/or blood transfusions in the 6 weeks prior to enrollment, allergy/intolerance to iron and inability to perform six-minutes walking test (6MWT).

A total of 74 patients met inclusion criteria and were divided into two groups:

- Group I:35 patients treated with FG (dose: $125 \mathrm{mg} /$ die IV);

- Group II:39 patients treated with FCM (maximum dose: 1000 $\mathrm{mg} /$ week IV).

FCM before 2018 was not available in our center, therefore patients enrolled in 2017 had been treated with FG; those enrolled in 2018 with FCM.

The following variables have been collected and stored in a centralized database in a de-identified manner for both groups of patients:

- Demographics and baseline anthropometric and clinical data (date of birth, height, weight, body mass index; co-morbidities such as diabetes, chronic obstructive pulmonary disease, chronic renal failure as defined by estimated glomerular filtration rate (calculated with MDRD formula) [eGFR] $<60 \mathrm{ml} / \mathrm{min} / 1.73 \mathrm{~m}^{3}$ ); pharmacological treatment; LVEF; in-hospital length-of-stay); Biochemical data (NT-proBNP, hemoglobin, serum iron, transferrin and ferritin levels and TSAT), both at admission and at discharge (after iron replacement therapy).

All patients underwent 6MWT both at admission and at discharge to assess exercise capacity.

Efficacy measures included changes from baseline in $\mathrm{Hb}$ and in distance traveled at 6MWT.

\section{Statistical analysis}

Statistical analysis was performed using SAS JMP v.10 software package. Continuous variables are described as mean \pm standard deviation, or as median and interquartile range, in case of nonnormal distribution. Categorical variables are expressed as number (percentages). Differences between groups were assessed by Student $t$-test, analysis of variance, or $x^{2}$-test, as appropriate. Contribution of baseline characteristics to study outcomes was assessed by univariate analysis and multivariable analysis. All derived cutoff values used for continuous variables have been chosen by means of receiver operating characteristic (ROC) curve analysis. Changes from baseline were tested by paired $t$-test or McNemar test, as appropriate. A p-value $<0.05$ was considered statistically significant; all tests were 2 -tailed.

\section{Results}

Data from 74 patients (mean age $67.5 \pm 10.4$ years, $43 \%$ women) were analyzed. Table 1 summarizes clinical and biochemical characteristics of the study population. No significant differences in demographics, drug therapy, type of surgery, LVEF and martial panel were observed between the 2 groups. Mean in-hospital length of treatment was $22.8 \pm 9.3$ days. At baseline, patients treated with FCM had higher prevalence of chronic renal failure (59\% vs 28\%, $\mathrm{p}=0.01$ ) and higher levels of NT-proBNP (3848 pg/ml vs $1335 \mathrm{pg} / \mathrm{ml}, \mathrm{p}=0.006$ ); no significant difference in functional capacity at admission was observed $(176.8 \pm 114$ meters in FCM group vs $173.7 \pm 73$ meters in FG group; $\mathrm{p}=0.88$ ).

Patients treated with FCM had significantly lower in-hospital length-of-stay (20.3 \pm 7 s $25.3 \pm 11.7$ days, $\mathrm{p}=0.043)$, greater distance walked at $6 \mathrm{MWT}$ at discharge than patients treated with FG (279.2 \pm 108.8 meters $v s 236.3 \pm 72.7$ meters, $\mathrm{p}=0.048)$ and higher $\Delta$ distance $(102.4 \pm 63$ vs 62.6 \pm 48.8 meters, $\mathrm{p}=0.003)$ (Figure 1, Table 2). At discharge, FCM-treated patients had lower NT-ProBNP levels (945 pg/ml vs $1272 \mathrm{pg} / \mathrm{ml}, \mathrm{p}=0.10)$, higher Hb levels $(11.1 \pm 1.2 \mathrm{~g} / \mathrm{dl}$ vs $10.2 \pm 1.1 \mathrm{~g} / \mathrm{dl}, \mathrm{p}=0.001)$ (Figure 2, Table 2), and significant $(>1$ $\mathrm{g} / \mathrm{dl}$ ) changes in Hb levels $(\mathrm{p}<0.0001)$ as compared to FG group.

After adjusting for age, sex and eGFR, multivariate analysis showed that transferrin levels [1.01 (1.00-1.02) $\mathrm{p}=0.028]$ and replacement therapy with FCM [8.22 (2.66-29.11), $\mathrm{p}<0.0002]$ were the most powerful predictors of $\mathrm{Hb}>1 \mathrm{~g} / \mathrm{dl}$ at discharge. Finally, FCM replacement therapy and iron serum levels were the most important predictors of 6MWT distance improvement ( $>100$ meters) at discharge (OR: 3.95, 1.31-13.06, $\mathrm{p}=0.013$ and OR: 0.92, 0.86$0.97, \mathrm{p}=0.003$, respectively).

\section{Discussion}

Cardiac rehabilitation is the best strategy approaching pre and post cardiac surgery $[12,13]$. In cardiac rehabilitation setting, anemia is commonly diagnosed after cardiac surgery and is often associated whit ID [14]. It is well known that anemia due to ID impairs $\mathrm{O}_{2}$ carrying and tissue oxidative capacity, resulting in a diminished peak $\mathrm{O}_{2}$ consumption and ability to endure submaximal exertion. Even in absence of anemia, ID can attenuate exercise performance [15].

ID is a treatable condition; however, it is still under-diagnosed and under-treated in clinical practice [16]. 
Several studies [4,17] demonstrated that IV iron loading improved exercise capacity and symptoms in patients with CHF and evidence of abnormal iron metabolism. In CHF, iron sucrose [18,19] and FCM [4] are the most adopted IV formulations for replacement therapy. Oral iron formulation is usually the first-line strategy; however, these supplementations may not be useful in all patients with IDA, since it is often poorly absorbed $[4,20]$. A recent study suggests that FCM is an effective therapy in patients with IDA who have gastrointestinal disorders and has a safety profile comparable to other IV iron agents [21].

We conducted a single-center retrospective study of anemic patients after cardiac surgery and found that those treated with FCM had shorter in-hospital stay, greater improvement in $\mathrm{Hb}$ values and in distance walked at 6MWT than patients treated with FG. To the

Table 1. Clinical and biochemical characteristics of study population.

\begin{tabular}{|c|c|c|c|}
\hline & Ferric gluconate (n=35) & Ferric carboxymaltose $(\mathrm{n}=39)$ & $\mathbf{p}$ \\
\hline Age (years) & $67 \pm 11$ & $68 \pm 9$ & 0.55 \\
\hline Females, n (\%) & $12(34)$ & $20(51)$ & 0.16 \\
\hline BMI, kg/m² & $28.8 \pm 6$ & $27.2 \pm 6$ & 0.25 \\
\hline CABG & $8(26)$ & $8(24)$ & 0.99 \\
\hline Valvular surgery & $23(74)$ & $25(75)$ & 0.99 \\
\hline Diabetes, n (\%) & $9(25)$ & $11(28)$ & 0.99 \\
\hline COPD, n (\%) & $9(25)$ & $8(20)$ & 0.78 \\
\hline Chronic renal failure, $\mathrm{n}(\%)$ & $10(28)$ & $23(59)$ & 0.01 \\
\hline Single antithrombotic therapy & $23(65)$ & $22(56)$ & 0.47 \\
\hline Double antithrombotic therapy & $1(3)$ & $2(5)$ & 0.99 \\
\hline Triple antithrombotic therapy & $0(0)$ & $1(2)$ & 0.99 \\
\hline Vitamin $\mathrm{K}$ antagonists & $19(54)$ & $19(48)$ & 0.64 \\
\hline NOACs & $4(11)$ & $8(20)$ & 0.35 \\
\hline LVEF, \% (IQR) & $60(48-60)$ & $60(55-60)$ & 0.33 \\
\hline NT-proBNP, ng/ml, median (range) & $1335(764-3157)$ & $3848(1147-7241)$ & 0.006 \\
\hline eGFR, $\mathrm{ml} / \mathrm{min} / 1.73 \mathrm{~m}^{3}$ & $71 \pm 21.8$ & $60.7 \pm 29.9$ & 0.08 \\
\hline $\mathrm{Hb}, \mathrm{g} / \mathrm{dl}$ & $9,5 \pm 1$ & $9,2 \pm 1$ & 0.19 \\
\hline Serum iron, $\mu g / d l$ & $35.5 \pm 8.2$ & $36.9 \pm 12.5$ & 0.58 \\
\hline Transferrin, mg/dl & $235 \pm 45$ & $227.5 \pm 57$ & 0.51 \\
\hline Ferritin, ng/ml & $375 \pm 283$ & $398 \pm 376.8$ & 0.76 \\
\hline Transferrin saturation, $\%$ & $10.4 \pm 2.8$ & $10.4 \pm 3.2$ & 0.92 \\
\hline In-hospital length-of-stay, days & $25.3 \pm 11.7$ & $20.3 \pm 7$ & 0.043 \\
\hline
\end{tabular}

BMI, body mass index; CABG, coronary artery bypass graft; COPD, chronic obstructive pulmonary disease; eGFR, estimated glomerular filtration rate; Hb, Hemoglobin; LVEF, left ventricular ejection fraction; NOACs, new oral anticoagulants; NT-proBNP, N-terminal pro-hormone of brain natriuretic peptide.

A



Ferric gluconate $\quad$ Ferric carboxymaltose
B $\quad \Delta$ Distance walked at six-minutes walking test at discharge

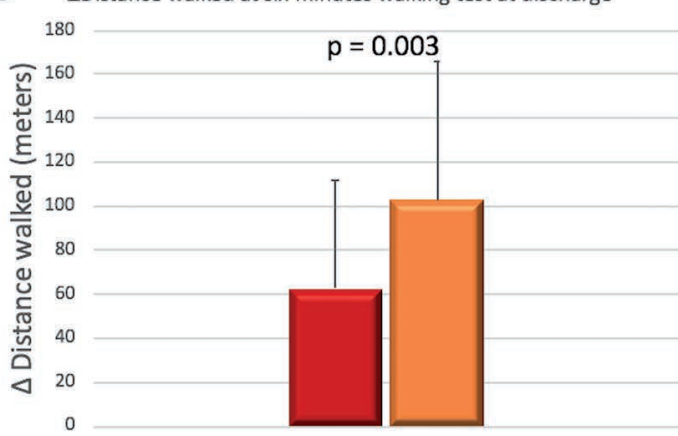

Ferric gluconate $\quad$ Ferric carboxymaltose

Figure 1. Distance walked at six-minutes walking test (A) and $\Delta$ distance at discharge (B) in patients treated with ferric gluconate and ferric carboxymaltose. 
best of our knowledge, this is the first study evaluating exercise capacity in patients without CHF and IDA after cardiac surgery.

In a large sample size population of consecutive routine elective surgical patients who were having major orthopedic surgery, abdominal, and genitourinary surgery, and other surgeries, Khalafallah et al .[22] found that postoperative IV FCM is a feasible and pragmatic management approach. More recently, in anemic patients undergoing elective cardiac surgery, Padmanabahn et al. [23] observed no significant differences in $\mathrm{Hb}$ increments after IV or oral iron administration; however, it should be remarked that only a small proportion of patients had proven ID. Notably, no adverse events were reported in all studies [22,23].

Previous studies showed encouraging evidences for iron replacement therapy in patients with CHF and ID [4]. Van Veldhuisen et al. [4] reported that treatment with intravenous FCM improves iron stores and showed a favorable effect on peak $\mathrm{VO}_{2}$. Ponikowsky et al. [24] found that treatment of symptomatic, irondeficient CHF patients treated with FCM for more than 1 year, had sustainable improvement in functional capacity, symptoms, and quality of life. Interestingly, Viethen et al. [25] reported that parenteral iron supplementation with FCM significantly improves exercise capacity and quality of life and is well tolerated in patients with pulmonary hypertension and iron deficiency. These data highlight that there is solid evidence suggesting that iron replacement therapy exerts beneficial effects on exercise capacity and is proven to be safe in HF patients with iron deficiency. However, type, dose and duration of replacement therapy as well as the effect on mortality and hospitalization still remain to be ascertained.

In the present study, we divided our population with IDA after cardiac surgery in two groups of IV iron replacement treatment. Mean ejection fraction in our population was normal. According to the literature, patients treated with FCM showed greater reduction of NT-proBNP levels [19]. In our population, levels of the NTproBNP at the baseline is greater in the FCM group, this data is probably correlated to lower eGFR that reflect a major neurohormonal activation. After treatment with FCM, we observed a significant reduction of NT-proBNP levels than FG group. We suppose that this result is correlated to the improvement of myocardial oxygenation and to the increase of $\mathrm{Hb}$ concentration that inhibiting the renin-angiotensin-aldosterone system reduce serum concentrations of NT-proBNP.

In the group treated with FCM we observed greater increase in hemoglobin values and in distance travelled at 6MWT at discharge. We did not observe any adverse events in both groups. Moreover, in our study, transferrin levels were predictors of increased hemoglobin on discharge. It is well known that transferrin contributed about as much to the model as transferrin saturation that predicts the probability of treatment response. This might explain the good discriminative power of transferrin in iron deficiency diagnosis.

Our results suggest that FCM may be an appropriate alternative to more established parenteral iron therapies, allowing higher doses with each infusion and therefore fewer infusions to achieve repletion of iron stores and rapid $\mathrm{Hb}$ responses. Furthermore, patients treated with FCM showed lower in-hospital rehabilitation length of stay. Use of FCM, reducing hospital stays, reduces the costs and risks associated with prolonged hospitalization.

Our results show that treatment of iron deficiency is crucial and FCM is effective in achieving this goal. However, further studies are mandatory in order to evaluate the effects of FCM replacement therapy on long-term outcomes in IDA patients after cardiac surgery.

\section{Study limitation}

This study has a number of limitations. First, this is a retrospective, single center study. Moreover, an important limitation of this study is the small sample size. Finally, we had no control group with IDA not treated or treated with oral iron replacement

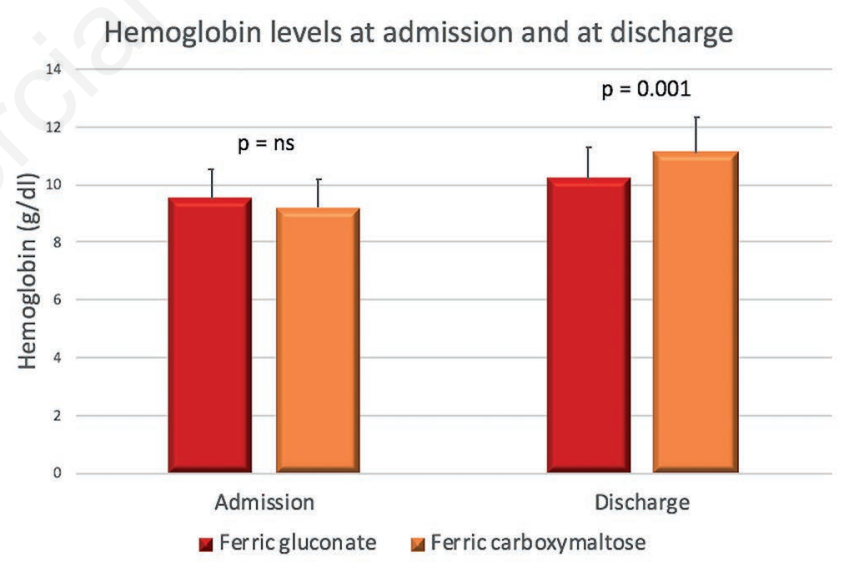

Figure 2. Hemoglobin levels at admission and at discharge in the two groups of patients.

Table 2. Hemoglobin and 6MWT parameters at discharge according to iron replacement therapy.

\begin{tabular}{lccc} 
& Ferric gluconate $(\mathrm{n=35})$ & Ferric carboxymaltose $(\mathrm{n}=39)$ & $\mathrm{p}$ \\
Distance at discharge (mt) & $236.3 \pm 72.7$ & $279.2 \pm 108.8$ & 0.048 \\
Distance at discharge (\%Predicted) & $50.9 \pm 15.7$ & $59.1 \pm 19.7$ & 0.052 \\
\hline$\Delta$ Distance at discharge & $+62.6 \pm 48.8$ & $+102.4 \pm 63$ & 0.003 \\
$\Delta$ Distance at discharge (\%Predicted) & $+13.8 \pm 11.3$ & $+22.12 \pm 14.7$ & 0.008 \\
\hline NT-ProBNP at discharge, median (range) & $1272(592-4146)$ & $945(487-1716)$ & 0.10 \\
$\mathrm{Hb}$ at discharge (g/dl) & $10.2 \pm 1.1$ & $11.1 \pm 1.2$ & 0.001 \\
$\Delta \mathrm{Hb}$ & $0.6 \pm 0.9$ & $1.9 \pm 1.1$ & $<0.0001$ \\
$\mathrm{Hb}>$ l at discharge $\mathrm{n}(\%)$ & $11(31)$ & $31(79)$ & $<0.0001$ \\
\hline
\end{tabular}

$\mathrm{Hb}$, hemoglobin. 
therapy, because in our center all patients with IDA are treated with intravenous iron replacement therapy according to guidelines.

\section{Conclusions}

Diagnosis and treatment of iron deficiency anemia in adult surgical patients is important in order to improve outcomes in a costeffective manner. Intravenous ferric carboxymaltose may be an effective therapy in this setting of patients and may improve exercise capacity after cardiac surgery. Further studies are necessary to confirm our preliminary data.

\section{References}

1. Najafi M, Faraoni D. Hemoglobin optimization and transfusion strategies in patients undergoing cardiac surgery. World J Cardiol 2015;7:377-82.

2. Musallam KM, Tamin HM, Richards T, et al. Preoperative anaemia and postoperative outcomes in non-cardiac surgery: a retrospective cohort study. Lancet 2011;378:1396-407.

3. Loor G, Koch CG, Sabik JF III, et al. Implications and management of anemia in cardiac surgery: current state of knowledge. J Thorac Cardiovasc Surg 2012;144: 538-46.

4. Van Veldhuisen DJ, Ponikowski P, van der Meer P, et al. Effect of ferric carboxymaltose on exercise capacity in patients with chronic heart failure and iron deficiency. Circulation 2017;136):1374-83.

5. Jankowska EA, von Haehling S, Anker SD, et al. Iron deficiency and heart failure: diagnostic dilemmas and therapeutic perspectives. Eur Heart J 2013;34:816-29.

6. Okonko DO, Mandal AK, Missouris CG, Poole-Wilson PA. Disordered iron homeostasis in chronic heart failure: prevalence, predictors, and relation to anemia, exercise capacity, and survival. J Am Coll Cardiol 2011;58:1241-51.

7. Grote Beverborg N, Klip IT, Meijers WC, et al. Definition of iron deficiency based on the gold standard of bone marrow iron staining in heart failure patients. Circ Heart Fail 2018;11:e004519.

8. Hass JD, Brownlie $\mathrm{T} 4^{\text {th }}$. Iron deficiency and reduced work capacity. J Nutr 2001;131:S676-88.

9. Andrews NC. Disorders of iron metabolism. N Eng J Med 1999;341:1986-95.

10. Murphy GJ, Reeves BC, Rogers CA, et al. Increased mortality, postoperative morbidity, and cost after red blood cell transfusion in patients having cardiac surgery. Circulation 2007;116:254452

11. Karkouti K, Wijeysundera DN, Yau TM, et al. Advance targeted transfusion in anemic cardiac surgical patients for kidney protection: an unblinded randomized pilot clinical trial. Anesthesiology 2012;116:613-21.

12. Pedretti RFE, Fattirolli F, Griffo R, et al. Cardiac Prevention and Rehabilitation "3.0": From acute to chronic phase. Position Paper of the Italian Association for Cardiovascular Prevention and Rehabilitation (GICR-IACPR). Monaldi Arch Chest Dis 2018;88:1004

13. Doyle MP, Indraratna P, Tardo DT, et al. Safety and efficacy of aerobic exercise commenced early after cardiac surgery: A systematic review and meta-analysis. Eur J Prev Cardiol 2019;26:36-45.

14. Tramarin R, Pistuddi V, Maresca L, et al. Patterns and determinants of functional and absolute iron deficiency in patients undergoing cardiac rehabilitation following heart surgery. Eur J Prev Cardiol 2017;24:799-807.

15. Pozzo J, Fournier P, Delmas C, et al. Absolute iron deficiency without anaemia in patients with chronic systolic heart failure is associated with poorer functional capacity. Arch Cardiovasc Dis 2017;110:99-105.

16. $\mathrm{Ng} \mathrm{O}$, Keeler BD, Mishra A, et al. Iron therapy for pre-operative anaemia. Cochrane Database Syst Rev 2015;12:CD011588.

17. Miñana G, Cardells I, Palau P, et al. Changes in myocardial iron content following administration of intravenous iron (Myocardial-IRON): Study design. Clin Cardiol 2018;41:72935.

18. Rocha BML, Cunha GJL, Menezes Falcão LF, et al. The burden of iron deficiency in heart failure: Therapeutic Approach. J Am Coll Cardiol 2018;71:782-93.

19. Toblli JE, Lombrana A, Duarte P, et al. Intravenous iron reduced NT-pro-brain natriuretic peptide in anemic patients with chronic heart failure and renal insufficiency. J Am Coll Cardiol 2007;50:1657-65.

20. Lewis GD, Malhotra R, Hernandez AF, et al. Effect of oral iron repletion on exercise capacity in patients with heart failure with reduced ejection fraction and iron deficiency: The IRONOUT HF randomized clinical trial. JAMA 2017;317:1958-66. Erratum in: JAMA 2017;317:2453.

21. Lichtenstein GR, Onken JE. Improved haemoglobin response with ferric carboxymaltose in patients with gastrointestinalrelated iron-deficiency anemia versus oral iron. Dig Dis Sci 2018;63:3009-19.

22. Khalafallah AA, Yan C, Al-Badri R, Robinson E, et al. Intravenous ferric carboxymaltose versus standard care in the management of postoperative anaemia: a prospective, openlabel, randomised controlled trial. Lancet Haematol 2016;3:e415-25.

23. Ponikowski P, van Veldhuisen DJ, Comin-Colet J, et al. Beneficial effects of long-term intravenous iron therapy with ferriccarboxymaltose in patients with symptomatic heart failure and iron deficiency. Eur Heart J 2015;36:657-68.

24. Padmanabhan H, Siau K, Nevill AM, et al. Intravenous iron does not effectively correct preoperative anaemia in cardiac surgery: a pilot randomized controlled trial. Interact Cardiovasc Thorac Surg 2018;28:447-54.

25. Viethen T, Gerhardt F, Dumitrescu D, et al. Ferric carboxymaltose improves exercise capacity and quality of life in patients with pulmonary arterial hypertension and iron deficiency: a pilot study. Int J Cardiol 2014;175:233-9. 